\title{
Tri-Stable Structural Switching in 2D Molecular Assembly at the Liquid/Solid Interface Triggered by External Electric Field
}

Shu-Ying Li, ${ }^{a, b}$ Xue-Qing Yang, ${ }^{a, c}$ Ting Chen, a * Dong Wang, ${ }^{a, d}$ Sheng-Fu Wang, ${ }^{c}$ and Li-Jun Wan ${ }^{a}$

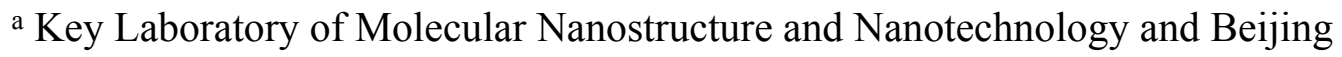
National Laboratory for Molecular Sciences, Institute of Chemistry, Chinese Academy of Sciences (CAS), Beijing 100190, P.R. China

${ }^{\mathrm{b}}$ Faculty of Chemistry, Northeast Normal University, Changchun 130024, P. R. China.

c Hubei University, Wuhan 400062, P. R. China.

d University of Chinese Academy of Sciences, Beijing 100049, P. R. China.

* To whom correspondence should be addressed: (Ting Chen) chenting@iccas.ac.cn 


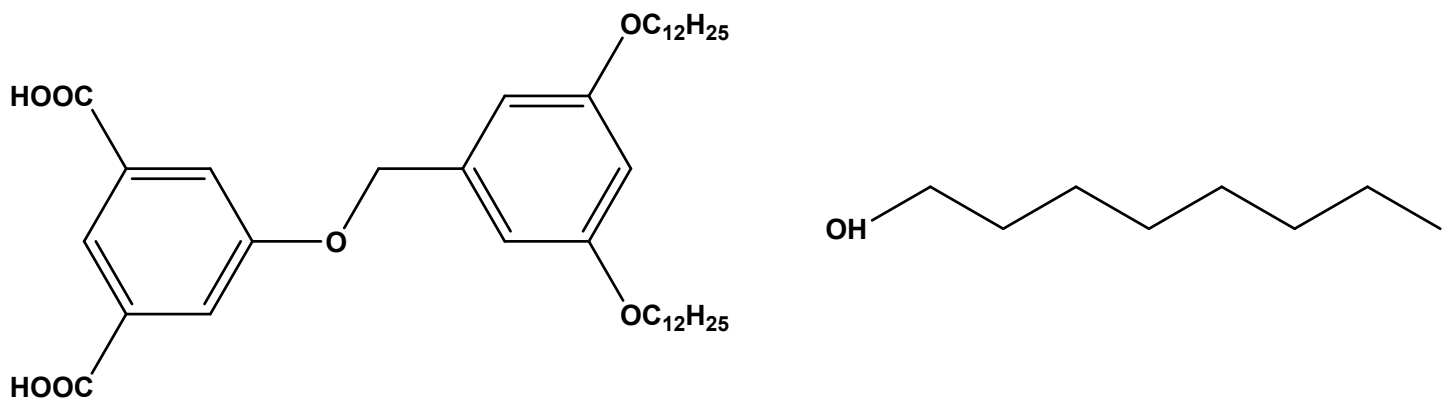

BIC-C12

1-octanol

Figure S1. Chemical structure of BIC-C12 and 1-octanol.
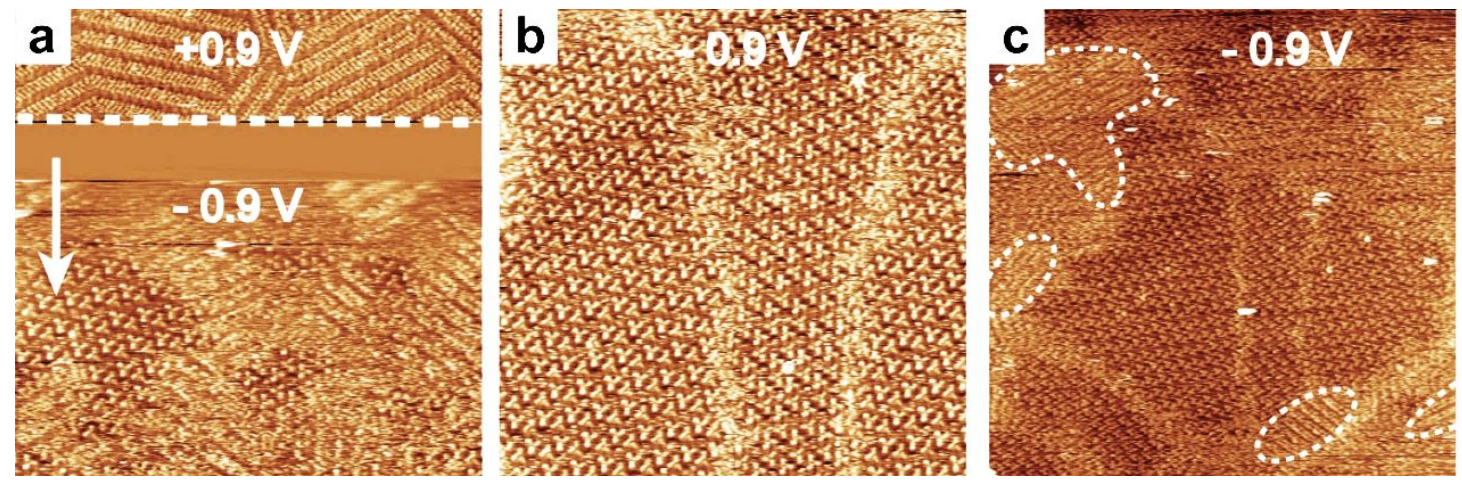

Figure S2. A series of STM images revealing the electric field induced structural switching is localized at the area around the STM tip. (a, b) STM images showing the structural switching from the lamellar structure to the honeycomb structure. (c) Zoom-out STM image recorded at the same position. Scan size: (a, b) $100 \mathrm{~nm} \times 100$ $\mathrm{nm}$, (c) $200 \mathrm{~nm} \times 200 \mathrm{~nm} . I=0.3 \mathrm{nA}$. 

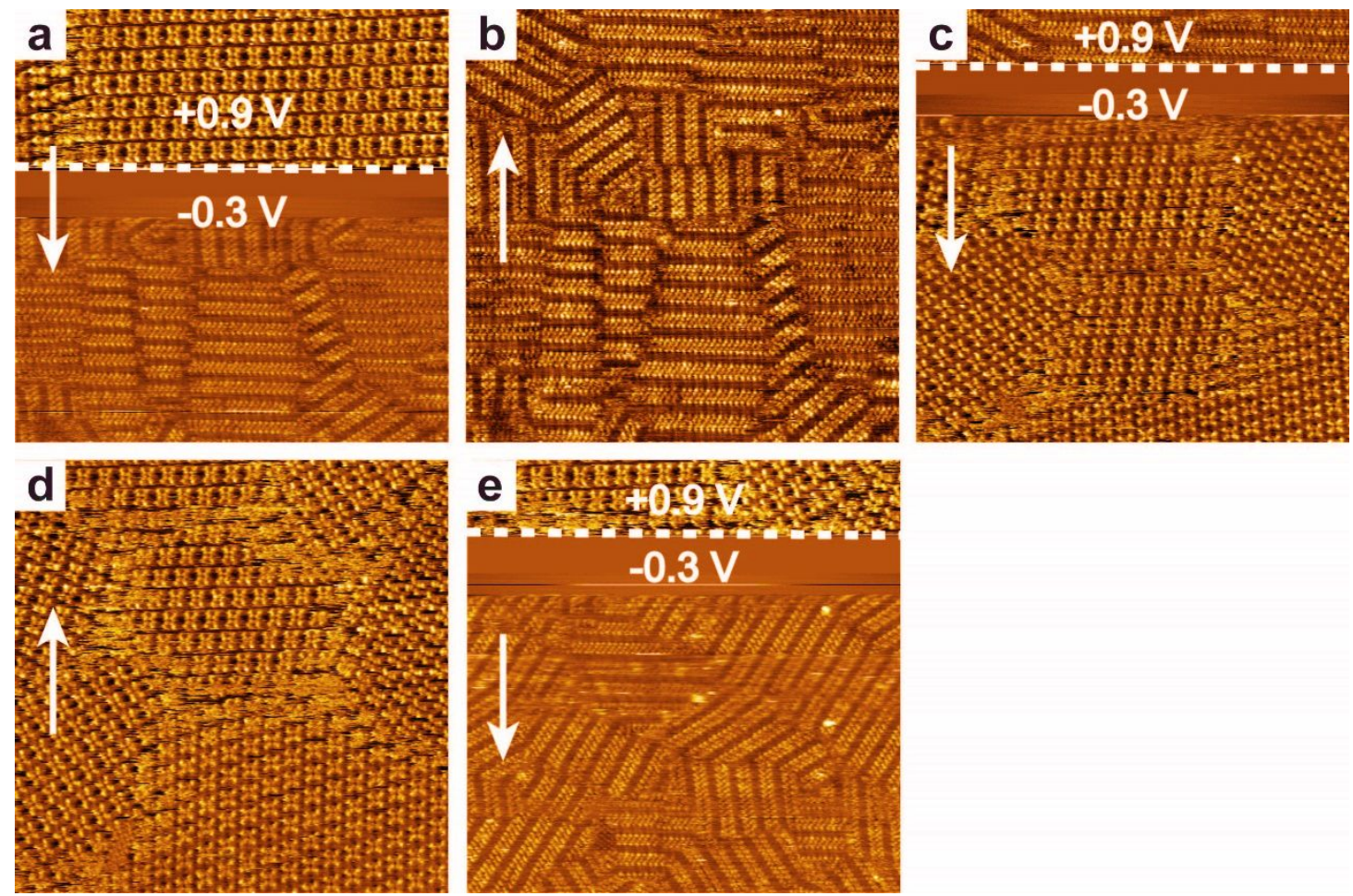

Figure S3. Sequential STM images showing the repeatability of the structural transition between the quadrangular structure and the lamellar structure. The concentration of BIC-C12 is $5 \times 10^{-5} \mathrm{~mol} \mathrm{~L}^{-1} . I=0.6 \mathrm{nA}$.
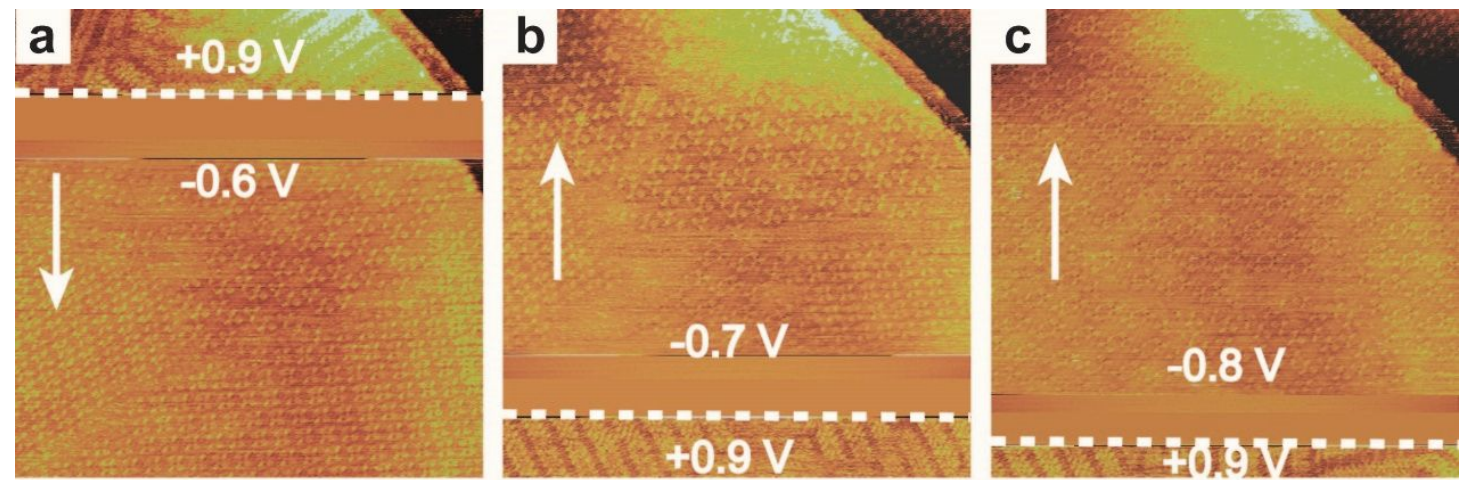

Figure S4. Typical STM images revealing structural transition induced by changing the sample bias from $+0.9 \mathrm{~V}$ to (a) $-0.6 \mathrm{~V}$, (b) $-0.7 \mathrm{~V}$, and (c) $-0.8 \mathrm{~V}$. The concentration of BIC-C12 is $5 \times 10^{-5} \mathrm{~mol} \mathrm{~L}^{-1} . I=0.6 \mathrm{nA}$. 


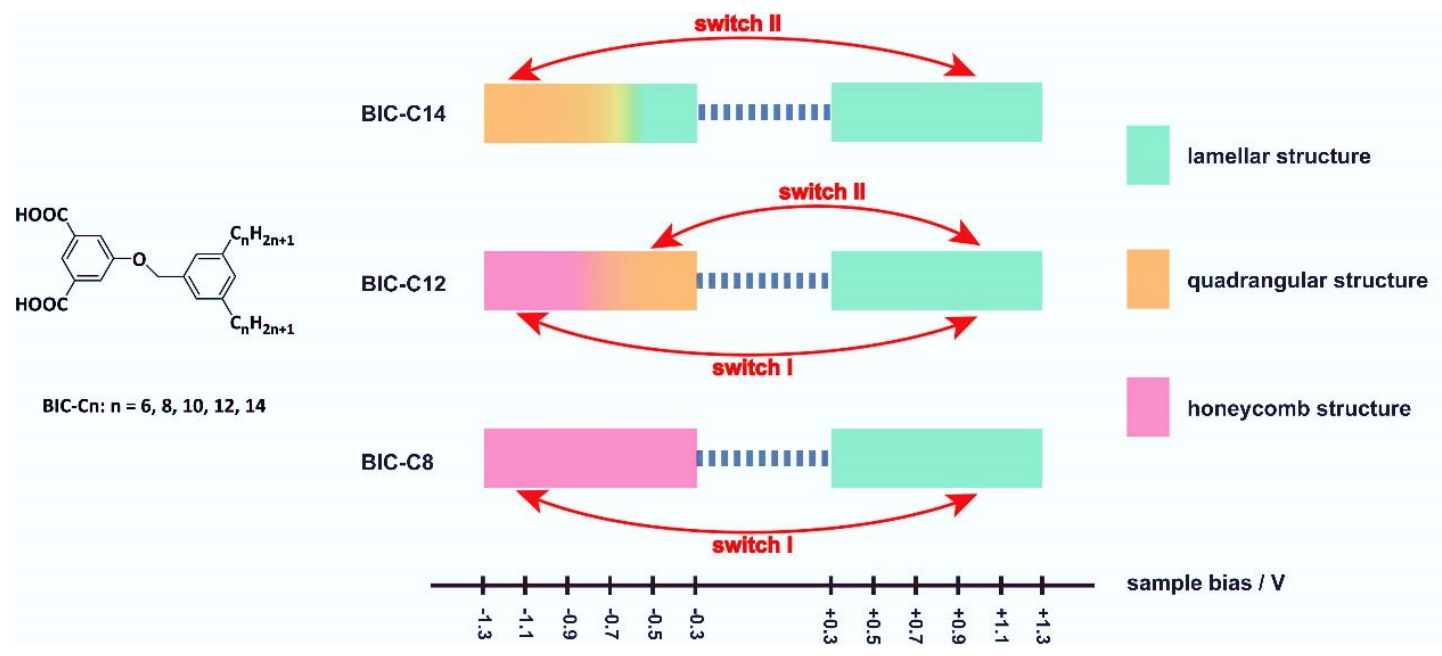

Figure S5. Chemical structures of BIC analogues and illustration of the response of the BIC analogue assemblies to the sample bias of STM setup.
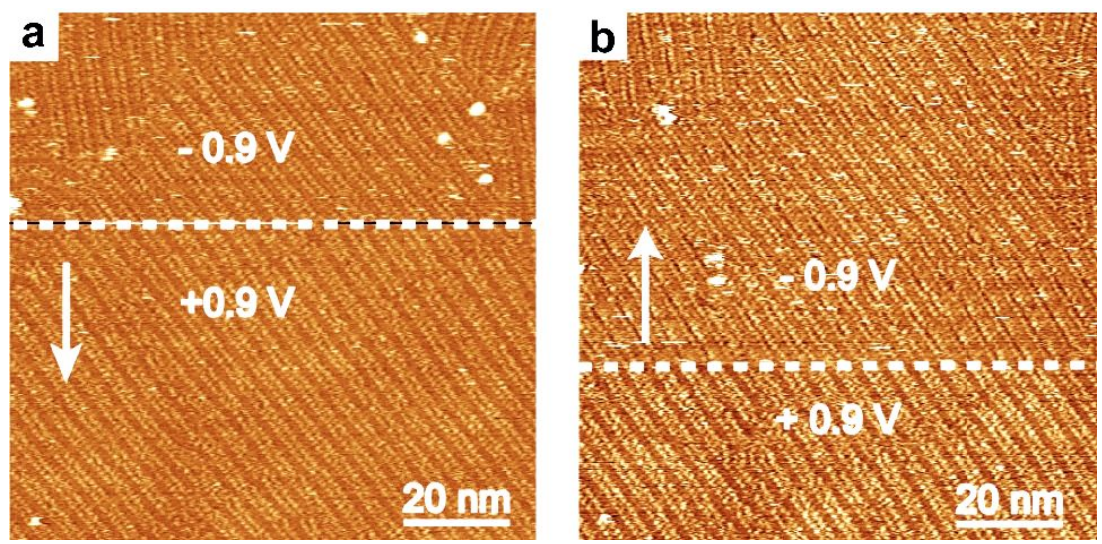

Figure S6. Typical STM images showing the BIC-C12 assembly at the air/HOPG interface is quite stable during switching of the sample bias between $-0.9 \mathrm{~V}$ and +0.9 $\mathrm{V}$. The concentration of BIC-C12 is $5 \times 10^{-5} \mathrm{~mol} \mathrm{~L}^{-1} . I=0.3 \mathrm{nA}$. 